\title{
COMPLETING BLOCK HERMITIAN MATRICES WITH MAXIMAL AND MINIMAL RANKS AND INERTIAS*
}

\author{
YONGGE TIAN ${ }^{\dagger}$
}

\begin{abstract}
For a Hermitian matrix with its main block diagonal given, this paper shows how to choose the off-diagonal blocks such that the resulting matrix has the maximal and minimal possible ranks and inertias, respectively. Some direct consequences and applications are also given.
\end{abstract}

Key words. Hermitian matrix, partial matrix, rank, inertia, matrix completion, matrix decomposition.

AMS subject classifications. 15A03, 15A23, 15B57.

1. Introduction. Throughout this paper, $\mathbb{C}^{m \times n}$ and $\mathbb{C}_{h}^{m \times m}$ denote the sets of all $m \times n$ complex matrices and $m \times m$ complex Hermitian matrices, respectively. The symbols $A^{T}, A^{*}$ and $r(A)$ stand for the transpose, conjugate transpose, and rank of a matrix $A \in \mathbb{C}^{m \times n}$, respectively; $I_{m}$ denotes the identity matrix of order $m$. We write $A \geq 0(A>0)$ if $A$ is Hermitian nonnegative (positive) definite. Two Hermitian matrices $A$ and $B$ of the same size are said to satisfy the (strict) Löwner partial ordering, denoted by $A \geq B(A>B)$, if $A-B$ is nonnegative (positive) definite.

As is well known, the eigenvalues of a Hermitian matrix $A \in \mathbb{C}_{h}^{m \times m}$ are all real, and the inertia of $A$ is defined to be the triplet

$$
\operatorname{In}(A)=\left\{i_{+}(A), i_{-}(A), i_{0}(A)\right\}
$$

where $i_{+}(A), i_{-}(A)$ and $i_{0}(A)$ are the numbers of the positive, negative and zero eigenvalues of $A$ counted with multiplicities, respectively. The two numbers $i_{+}(A)$ and $i_{-}(A)$ are usually called the positive index and negative index of inertia, or the positive and negative signatures, respectively. For a matrix $A \in \mathbb{C}_{h}^{m \times m}$, we have

$$
r(A)=i_{+}(A)+i_{-}(A), \quad i_{0}(A)=m-r(A) .
$$

Hence once $i_{+}(A)$ and $i_{-}(A)$ are both determined, $r(A)$ and $i_{0}(A)$ are both obtained as well.

\footnotetext{
${ }^{*}$ Received by the editors on June 15, 2009. Accepted for publication on July 31, 2010. Handling Editors: Roger A. Horn and Fuzhen Zhang.

$\dagger$ China Economics and Management Academy, Central University of Finance and Economics, Beijing 100081, China (yongge.tian@gmail.com).
} 
A partially specified block matrix is a matrix in which some submatrices are specified, while the remaining unspecified submatrices are free to be chosen from some matrix sets. One of the typical forms of partially specified block Hermitian matrices is given by

$$
M=\left[\begin{array}{cccc}
A_{11} & ? & \ldots & ? \\
?^{*} & A_{22} & \ldots & ? \\
\vdots & \vdots & \ddots & \vdots \\
?^{*} & ?^{*} & \ldots & A_{n n}
\end{array}\right]
$$

where $A_{j j} \in \mathbb{C}_{h}^{s_{j} \times s_{j}}$ are given, $j=1, \ldots, n$, and the symbols ?s and ?*s in the off-diagonal positions denote unspecified blocks (variable submatrices) and their conjugate transposes. Equation (1.2) is usually called a block diagonal partial Hermitian matrix. When the variable submatrices run over $\mathbb{C}^{s_{j} \times s_{k}}$, the matrix $M$ in (1.2), as well as its rank, range, nullity, inertia and so on also vary with respect to the choices of these variable submatrices. In this case, it would be of interest to complete the partial block matrix such that the resulting one has some prescribed properties. These kind of problems are usually called matrix completion problems in the literature. Matrix completion problems are a wide broad area in matrix theory and applications, which have attracted much attention since 1980s. Some previous work on determinant, rank, inertia, eigenvalue completions of partial matrices, as well as (skew)-Hermitian, positive (negative)-definite, invertible and inverse completions of partial matrices can be found, e.g., in [5]-[10], [15], [16], [19], [23], [24] and [28]-[35]. It has been noticed that completing a partial matrix with a prescribed property has a deep connections with computational complexity. Under different settings of variable matrices or unknown entries in the given matrix expressions or partial matrices, the problem is now known as P, RP, or NP-hard; see, e.g., [15], [20], [25] and [27].

Note that the inertia of a Hermitian matrix divides the eigenvalues of the matrix into three parts on the real line. Hence the inertia of a Hermitian matrix can be used to characterize definiteness of the matrix. The following results follow from the definitions of the rank and inertia of a (Hermitian) matrix.

Lemma 1.1. Let $A \in \mathbb{C}^{m \times m}, B \in \mathbb{C}^{m \times n}$, and $C \in \mathbb{C}_{h}^{m \times m}$. Then,

(a) $A$ is nonsingular if and only if $r(A)=m$.

(b) $B=0$ if and only if $r(B)=0$.

(c) $C>0(C<0)$ if and only if $i_{+}(C)=m\left(i_{-}(C)=m\right)$.

(d) $C \geq 0(C \leq 0)$ if and only if $i_{-}(C)=0\left(i_{+}(C)=0\right)$.

This lemma shows that if some formulas for ranks and inertias of Hermitian matrices are derived, then we can use them to characterize equalities and inequalities for Hermitian matrices. This basic algebraic method, which we refer to as the matrix 
rank/inertia method, is also available for approaching rank and inertia completion problems of partial matrices described above. Note that the rank and inertia of a Hermitian are all finite nonnegative integers. Hence the maximal and minimal values of the rank and inertia of a partial Hermitian matrix do exist with respect to the choices of the unspecified entries in it.

The aim of this paper is to derive explicit formulas for the maximal and minimal values of the rank and the positive and negative signatures of the matrix $M$ in (1.2) with respect to its unspecified blocks, and to determine at same time the unspecified blocks in (1.2) such that $M$ attains the extremal values. To do so, we need to use the following simple or well-known results on inertias of Hermitian matrices.

Lemma 1.2. Let $A \in \mathbb{C}_{h}^{m \times m}, B \in \mathbb{C}_{h}^{n \times n}, C \in \mathbb{C}^{m \times n}$, and assume that $P \in \mathbb{C}^{m \times m}$ is nonsingular. Then

$$
\begin{aligned}
i_{ \pm}\left(P A P^{*}\right) & =i_{ \pm}(A), \\
i_{ \pm}\left(A^{-1}\right) & =i_{ \pm}(A), \\
i_{ \pm}(\lambda A) & = \begin{cases}i_{ \pm}(A) & \text { if } \lambda>0 \\
i_{\mp}(A) & \text { if } \lambda<0,\end{cases} \\
i_{ \pm}\left[\begin{array}{cc}
A & I_{m} \\
I_{m} & 0
\end{array}\right] & =m, \\
i_{ \pm}\left[\begin{array}{cc}
A & 0 \\
0 & B
\end{array}\right] & =i_{ \pm}(A)+i_{ \pm}(B), \\
i_{ \pm}\left[\begin{array}{cc}
A & C \\
C^{*} & B
\end{array}\right] & \geq \max \left\{i_{ \pm}(A), i_{ \pm}(B)\right\}, \\
i_{ \pm}\left[\begin{array}{cc}
A & C \\
C^{*} & B
\end{array}\right] & =i_{ \pm}(A)+i_{ \pm}\left(B-C^{*} A^{-1} C\right), \text { if } A \text { is nonsingular. }
\end{aligned}
$$

Equation (1.3) are the well-known Sylvester's law of inertia. Equation (1.4) follows from the fact that the eigenvalues $A^{-1}$ is the reciprocals of those of $A$. Equation (1.5) is from the fact that the eigenvalues of $\lambda A$ are the eigenvalues of $A$ multiplied by $\lambda$. Equation (1.6) is from Lemma 1 in [22]. Equation (1.7) is from the definition of inertia. Equation (1.8) is the well-known Poincaré's inequality; see [11, 21]. Equation (1.9) is well known; see Theorem 1 in [21].

2. Rank and inertia completions for a $2 \times 2$ block Hermitian matrix. We first consider the block Hermitian matrix in (1.2) for $n=2$ :

$$
M(X)=\left[\begin{array}{cc}
A & X \\
X^{*} & B
\end{array}\right]
$$


where $A \in \mathbb{C}_{h}^{m \times m}, B \in \mathbb{C}_{h}^{n \times n}$ and $X \in \mathbb{C}^{m \times n}$. Equation (2.1) is a standard form of block Hermitian matrix in the investigations of Hermitian matrices and their applications. Equalities and inequalities for the rank and inertia of the block Hermitian matrix in (2.1), as well as various completion problems associated with its rank and inertia were widely studied in the literature; see, e.g., [2]-[5], [8], [11]-[14], [17], [18] and [26]. Also note that the $M(X)$ in (2.1) can be rewritten as

$$
M(X)=\left[\begin{array}{cc}
A & 0 \\
0 & B
\end{array}\right]+\left[\begin{array}{c}
I_{m} \\
0
\end{array}\right] X\left[0, I_{n}\right]+\left[\begin{array}{c}
0 \\
I_{n}
\end{array}\right] X^{*}\left[I_{m}, 0\right] .
$$

Hence the rank and inertia of $M(X)$ can also be derived from the general results on the rank and inertia of the matrix expression $A-B X C-(B X C)^{*}$ given in the recent paper [4].

In this section, we revisit the rank and inertia of $M(X)$ in (2.1) through the canonical forms of $A$ and $B$ under *-congruence transformation. We shall use the canonical forms to derive the maximal and minimal values of the rank and inertia of the matrix $M(X)$ in (2.1), and give the procedure of choosing $X \mathrm{~s}$ such that the rank and inertia of $M(X)$ attain the extremal values respectively.

TheOrem 2.1. Let $M(X)$ be as given in (2.1). Then

$$
\begin{aligned}
\max _{X \in \mathbb{C}^{m \times n}} r[M(X)] & =\min \{m+n, \quad r(A)+2 n, \quad r(B)+2 m\}, \\
\min _{X \in \mathbb{C}^{m \times n}} r[M(X)] & =\max \left\{r(A), \quad r(B), \quad i_{+}(A)+i_{-}(B), \quad i_{-}(A)+i_{+}(B)\right\}, \\
\max _{X \in \mathbb{C}^{m \times n}} i_{ \pm}[M(X)] & =\min \left\{i_{ \pm}(A)+n, \quad i_{ \pm}(B)+m\right\}, \\
\min _{X \in \mathbb{C}^{m \times n}} i_{ \pm}[M(X)] & =\max \left\{i_{ \pm}(A), \quad i_{ \pm}(B)\right\} .
\end{aligned}
$$

Hence,

(a) There exists an $X \in \mathbb{C}^{m \times n}$ such that $M(X)$ is nonsingular if and only if $r(A) \geq m-n$ and $r(B) \geq n-m$.

(b) $M(X)$ is nonsingular for any $X \in \mathbb{C}^{m \times n}$ if and only if $A>0$ and $B<0$, or $A<0$ and $B>0$.

(c) There exists an $X \in \mathbb{C}^{m \times n}$ such that $M(X)>0(M(X)<0)$ if and only if $A>0$ and $B>0(A<0$ and $B<0)$.

(d) There exists an $X \in \mathbb{C}^{m \times n}$ such that $M(X) \geq 0(M(X) \leq 0)$ if and only if $A \geq 0$ and $B \geq 0(A \leq 0$ and $B \leq 0)$.

Proof. Since both $A$ and $B$ are Hermitian matrices, they can be decomposed under $*$-congruence transformation into the following canonical forms

$$
W_{1} A W_{1}^{*}=\operatorname{diag}\left(I_{p_{1}},-I_{q_{1}}, 0\right):=D_{1}, \quad W_{2} B W_{2}^{*}=\operatorname{diag}\left(0,-I_{q_{2}}, I_{p_{2}}\right):=D_{2},
$$


where $W_{1}$ and $W_{2}$ are two nonsingular matrices, $p_{1}=i_{+}(A), q_{1}=i_{-}(A), p_{2}=i_{+}(B)$ and $q_{2}=i_{-}(B)$. Denote $\widehat{X}=W_{1} X W_{2}^{*}$ and $W=\operatorname{diag}\left(W_{1}, W_{2}\right)$. Correspondingly, $W M(X) W^{*}$ can be written as

$$
W M(X) W^{*}=\left[\begin{array}{cc}
D_{1} & \widehat{X} \\
\widehat{X}^{*} & D_{2}
\end{array}\right]=\left[\begin{array}{cccccc}
I_{p_{1}} & 0 & 0 & \widehat{X}_{11} & \widehat{X}_{12} & \widehat{X}_{13} \\
0 & -I_{q_{1}} & 0 & \widehat{X}_{21} & \widehat{X}_{22} & \widehat{X}_{23} \\
0 & 0 & 0 & \widehat{X}_{31} & \widehat{X}_{32} & \widehat{X}_{33} \\
\widehat{X}_{11}^{*} & \widehat{X}_{21}^{*} & \widehat{X}_{31}^{*} & 0 & 0 & 0 \\
\widehat{X}_{12}^{*} & \widehat{X}_{22}^{*} & \widehat{X}_{32}^{*} & 0 & -I_{q_{2}} & 0 \\
\widehat{X}_{13}^{*} & \widehat{X}_{23}^{*} & \widehat{X}_{33}^{*} & 0 & 0 & I_{p_{2}}
\end{array}\right]:=\Phi .
$$

From (1.3), the inertia and rank of $M(X)$ in (2.1) satisfy

$$
i_{ \pm}[M(X)]=i_{ \pm}(\Phi) \text { and } r[M(X)]=r(\Phi) .
$$

Note from the dimension of $M(X)$ in (2.1) and the well-known rank inequality

$$
r(P+Q) \leq r(P)+r(Q)
$$

that the rank of $M(X)$ satisfies the inequalities $r[M(X)] \leq m+n$, and

$$
\begin{aligned}
& r[M(X)] \leq r(A)+r\left[\begin{array}{cc}
0 & X \\
X^{*} & B
\end{array}\right] \leq r(A)+2 n, \\
& r[M(X)] \leq r(B)+r\left[\begin{array}{cc}
A & X \\
X^{*} & 0
\end{array}\right] \leq r(B)+2 m .
\end{aligned}
$$

Combining them yields the following inequality

$$
r[M(X)] \leq \min \{m+n, \quad r(A)+2 n, \quad r(B)+2 m\} .
$$

We next choose the arbitrary matrix $\widehat{X}$ in (2.7) such that the upper bound in (2.9) is attained.

Case 1. Assume that $m+n \leq \min \{r(A)+2 n, r(B)+2 m\}$, and also assume $m \leq n$ without loss of generality. In this case, set $\widehat{X}=\left[t I_{m}, 0\right]$, where $t$ is a real number. Then

$$
r(\Phi)=r\left[\begin{array}{cc}
D_{1} & \widehat{X} \\
\widehat{X}^{*} & D_{2}
\end{array}\right]=r\left[\begin{array}{ccc}
D_{1} & t I_{m} & 0 \\
t I_{m} & D_{21} & 0 \\
0 & 0 & D_{22}
\end{array}\right]=r\left[\begin{array}{cc}
t I_{m} & D_{1} \\
D_{21} & t I_{m}
\end{array}\right]+r\left(D_{22}\right),
$$

where $D_{2}=\operatorname{diag}\left(D_{21}, D_{22}\right)$ with $r\left(D_{22}\right)=n-m$. Thus, there exists a (sufficiently large or small) real number $t$ such that $r\left[\begin{array}{cc}t I_{m} & D_{1} \\ D_{21} & t I_{m}\end{array}\right]=2 m$, so that $r[M(X)]=2 m+(n-m)=m+n$. 
Case 2. Assume that $r(A)+2 n \leq \min \{m+n, r(B)+2 m\}$. Then setting $\widehat{X}=$ $\left[0, t I_{n}\right]^{T}$ with $t \neq 0$ leads to

$$
r(\Phi)=r\left[\begin{array}{cc}
D_{1} & \widehat{X} \\
\widehat{X}^{*} & D_{2}
\end{array}\right]=r\left[\begin{array}{ccc}
D_{11} & 0 & 0 \\
0 & 0 & t I_{n} \\
0 & t I_{n} & D_{2}
\end{array}\right]=2 n+r\left(D_{11}\right),
$$

where $D_{1}=\operatorname{diag}\left(D_{11}, 0\right)$ with $r\left(D_{11}\right)=r(A)$, so that $r[M(X)]=r(A)+2 n$.

The case for $r(B)+2 m \leq \min \{m+n, r(A)+2 n\}$ can be shown similarly. Combining the three cases leads to $(2.2)$.

Recall that the rank of a matrix is always greater than or equal to the ranks of its submatrices. Hence it is easy to see from (2.7) and its submatrices that

$$
\begin{gathered}
r(\Phi) \geq r\left[\begin{array}{cc}
I_{p_{1}} & 0 \\
0 & -I_{q_{1}}
\end{array}\right]=p_{1}+q_{1}, r(\Phi) \geq r\left[\begin{array}{cc}
I_{p_{2}} & 0 \\
0 & -I_{q_{2}}
\end{array}\right]=p_{2}+q_{2}, \\
r(\Phi) \geq r\left[\begin{array}{cc}
I_{p_{1}} & \widehat{X}_{23} \\
\widehat{X}_{23}^{*} & -I_{q_{2}}
\end{array}\right]=r\left[\begin{array}{cc}
I_{p_{1}} & 0 \\
0 & -I_{q_{2}}-\widehat{X}_{23}^{*} \widehat{X}_{23}
\end{array}\right]=p_{1}+r\left(I_{q_{2}}+\widehat{X}_{23}^{*} \widehat{X}_{23}\right) \\
=p_{1}+q_{2}, \\
r(\Phi) \geq r\left[\begin{array}{cc}
-I_{q_{1}} & \widehat{X}_{21} \\
\widehat{X}_{21}^{*} & I_{p_{2}}
\end{array}\right]=r\left[\begin{array}{cc}
-I_{q_{1}} & 0 \\
0 & I_{p_{2}}+\widehat{X}_{21}^{*} \widehat{X}_{21}
\end{array}\right]=q_{1}+r\left(I_{p_{2}}+\widehat{X}_{21}^{*} \widehat{X}_{21}\right) \\
=q_{1}+p_{2},
\end{gathered}
$$

so that the inequality

$$
\begin{aligned}
r(\Phi) & \geq \max \left\{p_{1}+q_{1}, \quad p_{1}+q_{2}, \quad p_{2}+q_{1}, \quad p_{2}+q_{2}\right\} \\
& =\max \left\{p_{1}, \quad p_{2}\right\}+\max \left\{q_{1}, \quad q_{2}\right\}
\end{aligned}
$$

follows. We next choose the submatrices $\widehat{X}_{j k}$ in (2.7) such that the lower bound in (2.10) is attained.

Case 1. If $p_{1} \geq p_{2}$ and $q_{1} \geq q_{2}$, we choose $\widehat{X}_{12}=0, \widehat{X}_{13}=0, \widehat{X}_{21}=0, \widehat{X}_{23}=0$, $\widehat{X}_{31}=0, \widehat{X}_{32}=0, \widehat{X}_{33}=0, \widehat{X}_{11}=\left[I_{p_{2}}, 0\right]^{T}$ and $\widehat{X}_{22}=\left[I_{q_{2}}, 0\right]^{T}$. In this case, we have

$$
r[M(X)]=p_{1}+q_{1}=\max \left\{p_{1}, p_{2}\right\}+\max \left\{q_{1}, q_{2}\right\} .
$$

Case 2. If $p_{1} \geq p_{2}$ and $q_{1} \leq q_{2}$, then we choose $\widehat{X}_{12}=0, \widehat{X}_{13}=0, \widehat{X}_{21}=0, \widehat{X}_{23}=0$, $\widehat{X}_{31}=0, \widehat{X}_{32}=0, \widehat{X}_{33}=0, \widehat{X}_{11}=\left[I_{p_{2}}, 0\right]^{T}$ and $\widehat{X}_{22}=\left[I_{q_{1}}, 0\right]^{T}$. In this case, we have

$$
r[M(X)]=p_{1}+q_{2}=\max \left\{p_{1}, \quad p_{2}\right\}+\max \left\{q_{1}, \quad q_{2}\right\} .
$$


The remaining two cases for $p_{1} \leq p_{2}, q_{1} \geq q_{2}$ and $p_{1} \leq p_{2}, q_{1} \leq q_{2}$ can be shown similarly. Thus (2.3) follows.

To show (2.4), we set

$$
\widehat{X}=\left[\begin{array}{cc}
0 & 0 \\
t I_{m-p_{1}} & 0
\end{array}\right] \text { if } m-p_{1} \leq n-p_{2}, \text { and } \widehat{X}=\left[\begin{array}{cc}
0 & 0 \\
t I_{n-p_{2}} & 0
\end{array}\right] \text { if } m-p_{1} \geq n-p_{2}
$$

in (2.7). Also denote $J_{1}=\operatorname{diag}\left(-I_{q_{1}}, 0\right)$ and $J_{2}=\operatorname{diag}\left(0,-I_{q_{2}}\right)$. Then it can be derived from (1.6) and (1.7) that

$$
\begin{aligned}
& i_{+}(\Phi)=i_{+}\left[\begin{array}{cccc}
I_{p_{1}} & 0 & 0 & 0 \\
0 & J_{1} & {\left[t I_{m-p_{1}}, 0\right]} & 0 \\
0 & {\left[t I_{m-p_{1}}, 0\right]^{T}} & J_{2} & 0 \\
0 & 0 & 0 & I_{p_{2}}
\end{array}\right] \\
& =p_{1}+p_{2}+i_{+}\left[\begin{array}{cc}
J_{1} & {\left[t I_{m-p_{1}}, 0\right]} \\
{\left[t I_{m-p_{1}}, 0\right]^{T}} & J_{2}
\end{array}\right] \\
& =p_{1}+p_{2}+i_{+}\left[\begin{array}{cc}
J_{1} & t I_{m-p_{1}} \\
t I_{m-p_{1}} & 0
\end{array}\right] \\
& =p_{1}+p_{2}+m-p_{1}=m+p_{2} \text { if } m-p_{1} \leq n-p_{2} \text {, } \\
& i_{+}(\Phi)=i_{+}\left[\begin{array}{cccc}
I_{p_{1}} & 0 & 0 & 0 \\
0 & J_{1} & {\left[t I_{n-p_{2}}, 0\right]^{T}} & 0 \\
0 & {\left[t I_{n-p_{2}}, 0\right]} & J_{2} & 0 \\
0 & 0 & 0 & I_{p_{2}}
\end{array}\right] \\
& =p_{1}+p_{2}+i_{+}\left[\begin{array}{cc}
J_{1} & {\left[t I_{n-p_{2}}, 0\right]^{T}} \\
{\left[t I_{n-p_{2}}, 0\right]} & J_{2}
\end{array}\right] \\
& =p_{1}+p_{2}+i_{+}\left[\begin{array}{cc}
0 & t I_{n-p_{2}} \\
t I_{n-p_{2}} & J_{2}
\end{array}\right] \\
& =p_{1}+p_{2}+n-p_{2}=n+p_{1} \text { if } m-p_{1} \geq n-p_{2} \text {. }
\end{aligned}
$$

Combining (2.11) and (2.12) with (2.8) leads to (2.4) for the positive signature. Equation (2.4) for the negative signature can be shown similarly.

From (1.8), the right-hand sides of $(2.5)$ are lower bounds of $i_{ \pm}[M(X)]$. We next choose the matrix $X$ such that $i_{ \pm}[M(X)]$ are equal to the lower bounds. If $i_{+}(A) \leq i_{+}(B)$, we set $\widehat{X}=\left[\begin{array}{cc}0 & {\left[I_{p_{1}}, 0\right]} \\ 0 & 0\end{array}\right]$. Substituting it into (2.7) and applying (1.9) gives

$$
i_{+}(\Phi)=i_{+}\left[\begin{array}{cc}
I_{p_{1}} & {\left[I_{p_{1}}, 0\right]} \\
{\left[I_{p_{1}}, 0\right]^{T}} & I_{p_{2}}
\end{array}\right]=i_{+}\left[\begin{array}{cc}
0 & 0 \\
0 & I_{p_{2}}
\end{array}\right]=p_{2}
$$


If $i_{+}(A) \geq i_{+}(B)$, we set $\widehat{X}=\left[\begin{array}{cc}0 & {\left[I_{p_{2}}, 0\right]^{T}} \\ 0 & 0\end{array}\right]$. Substituting it into (2.7) and applying (1.9) gives

$$
i_{+}(\Phi)=i_{+}\left[\begin{array}{cc}
I_{p_{1}} & {\left[I_{p_{2}}, 0\right]^{T}} \\
{\left[I_{p_{2}}, 0\right]} & I_{p_{2}}
\end{array}\right]=i_{+}\left[\begin{array}{cc}
I_{p_{1}} & 0 \\
0 & 0
\end{array}\right]=p_{1} .
$$

Combining the two cases yields the formula in (2.5) for the positive signature. The formula in (2.5) for the negative signature can be shown similarly.

Results (a)-(d) follow from (2.2)-(2.5) and Lemma 1.1.

The proof of Theorem 2.1 is constructive, namely, procedures to choose a matrix $X$ that satisfies (2.2)-(2.5) respectively are given in the derivations of the extremal ranks and inertias.

The quantities on the right-hand sides of (2.2)-(2.5), as upper and lower bounds for the rank and inertia of $M(X)$ in (2.1), were given in the literature. For example, the right-hand sides of (2.4) and (2.5) were given in the first theorem of [2] as upper and lower bounds for the inertia of $M(X)$. But the matrices $X$ satisfying (2.4) and (2.5) were not given in [2].

Notice that Theorem 2.1 and its proof show how to choose the variable matrix $X$ such that the rank and inertia of $M(X)$ in (2.1) attain their maximal and minimal values. Hence we can easily use them, as demonstrated in Theorem 2.1(a)-(d), to study various invertible and definiteness completions of $M(X)$ in (2.1). Some previous work on invertible and nonnegative definite completions of partial Hermitian matrices can be found in $[12,14,19]$.

A variation of the block Hermitian matrix in (2.1) is given by

$$
M(X, Y)=\left[\begin{array}{cc}
A & X \\
X^{*} & Y
\end{array}\right]
$$

where $A \in \mathbb{C}_{h}^{m \times m}$ is given, and $X \in \mathbb{C}^{m \times n}$ and $Y \in \mathbb{C}_{h}^{n \times n}$ are two variable matrices. Some upper and lower bounds for the inertia of $M(X, Y)$ were given in Lemma 4.1 of [6]. From Theorem 2.1, we now can derive the maximal and minimal values of the ranks and inertias of $M(X, Y)$ in (2.13) with respect to the two variable matrices $X$ and $Y$. 
Corollary 2.2. Let $M(X, Y)$ be as given in (2.13). Then

$$
\begin{aligned}
\max _{X \in \mathbb{C}^{m \times n}, Y \in \mathbb{C}_{h}^{n \times n}} r[M(X, Y)] & =\min \{m+n, \quad r(A)+2 n\}, \\
\min _{X \in \mathbb{C}^{m \times n}, Y \in \mathbb{C}_{h}^{n \times n}} r[M(X, Y)] & =r(A), \\
\max _{X \in \mathbb{C}^{m \times n}, Y \in \mathbb{C}_{h}^{n \times n}} i_{ \pm}[M(X, Y)] & =n+i_{ \pm}(A), \\
\min _{X \in \mathbb{C}^{m \times n}, Y \in \mathbb{C}_{h}^{n \times n}} i_{ \pm}[M(X, Y)] & =i_{ \pm}(A) .
\end{aligned}
$$

Proof. We have

$$
\max _{B \in \mathbb{C}_{h}^{n \times n}} r(B)=n, \min _{B \in \mathbb{C}_{h}^{n \times n}} r(B)=0, \max _{B \in \mathbb{C}_{h}^{n \times n}} i_{ \pm}(B)=n, \min _{B \in \mathbb{C}_{h}^{n \times n}} i_{ \pm}(B)=0 .
$$

Substituting them into (2.2)-(2.5) leads to (2.14)-(2.17).

As a useful application of Theorem 2.1, we are now able to derive the maximal and minimal values of the rank and inertia of the simple quadratic Hermitian matrix expression $B-X^{*} A X$ with respect to a variable matrix $X$.

Theorem 2.3. Let $A \in \mathbb{C}_{h}^{m \times m}$ and $B \in \mathbb{C}_{h}^{n \times n}$ be given, and assume that $A$ is nonsingular. Then

$$
\begin{aligned}
& \min _{X \in \mathbb{C}^{m \times n}} r\left(B-X^{*} A X\right) \\
&=\max \left\{0, r(B)-m, i_{+}(B)-i_{+}(A), i_{-}(B)-i_{-}(A)\right\}, \\
& \max _{X \in \mathbb{C}^{m \times n}} i_{ \pm}\left(B-X^{*} A X\right)=\min \left\{n, i_{\mp}(A)+i_{ \pm}(B)\right\}, \\
& \min _{X \in \mathbb{C}^{m \times n}} i_{ \pm}\left(B-X^{*} A X\right)=\max \left\{0, i_{ \pm}(B)-i_{ \pm}(A)\right\} .
\end{aligned}
$$

Hence,

(a) There exists an $X \in \mathbb{C}^{m \times n}$ such that $B-X^{*} A X$ is nonsingular if and only if $r(B) \geq n-m$.

(b) There exists an $X \in \mathbb{C}^{m \times n}$ such that $X^{*} A X=B$ if and only if both $i_{+}(B) \leq$ $i_{+}(A)$ and $i_{-}(B) \leq i_{-}(A)$.

(c) There exists an $X \in \mathbb{C}^{m \times n}$ such that $B-X^{*} A X>0\left(B-X^{*} A X<0\right)$ if and only if $i_{-}(A)+i_{+}(B) \geq n\left(i_{+}(A)+i_{-}(B) \geq n\right)$. 
(d) $B-X^{*} A X>0\left(B-X^{*} A X<0\right)$ for all $X \in \mathbb{C}^{m \times n}$ if and only if both $A \leq 0$ and $B>0$ (both $A \geq 0$ and $B<0)$.

(e) There exists an $X \in \mathbb{C}^{m \times n}$ such that $B-X^{*} A X \geq 0\left(B-X^{*} A X \leq 0\right)$ if and only if $i_{-}(B) \leq i_{-}(A)\left(i_{+}(B) \leq i_{+}(A)\right)$.

(f) $B-X^{*} A X \geq 0\left(B-X^{*} A X \leq 0\right)$ for all $X \in \mathbb{C}^{m \times n}$ if and only if both $A \leq 0$ and $B \geq 0$ (both $A \geq 0$ and $B \leq 0)$. gives

Proof. Let $M=\left[\begin{array}{cc}A^{-1} & X \\ X^{*} & B\end{array}\right]$. Then applying (1.1), (1.4), and (1.9) to this $M$

$$
\begin{aligned}
i_{ \pm}(M) & =i_{ \pm}\left(A^{-1}\right)+i_{ \pm}\left(B-X^{*} A X\right)=i_{ \pm}(A)+i_{ \pm}\left(B-X^{*} A X\right), \\
r(M) & =m+r\left(B-X^{*} A X\right) .
\end{aligned}
$$

Hence we have

$$
\begin{aligned}
& \max _{X \in \mathbb{C}^{m \times n}} r(M)=m+\max _{X \in \mathbb{C}^{m \times n}} r\left(B-X^{*} A X\right), \\
& \min _{X \in \mathbb{C}^{m \times n}} r(M)=m+\min _{X \in \mathbb{C}^{m \times n}} r\left(B-X^{*} A X\right), \\
& \max _{X \in \mathbb{C}^{m \times n}} i_{ \pm}(M)=i_{ \pm}(A)+\max _{X \in \mathbb{C}^{m \times n}} i_{ \pm}\left(B-X^{*} A X\right), \\
& \min _{X \in \mathbb{C}^{m \times n}} i_{ \pm}(M)=i_{ \pm}(A)+\min _{X \in \mathbb{C}^{m \times n}} i_{ \pm}\left(B-X^{*} A X\right) .
\end{aligned}
$$

On the other hand, applying (2.2)-(2.5) to the $M$ also gives

$$
\begin{aligned}
\max _{X \in \mathbb{C}^{m \times n}} r(M) & =\min \{m+n, \quad r(B)+2 m\}, \\
\min _{X \in \mathbb{C}^{m \times n}} r(M) & =\max \left\{m, \quad r(B), \quad i_{+}(A)+i_{-}(B), \quad i_{-}(A)+i_{+}(B)\right\}, \\
\max _{X \in \mathbb{C}^{m \times n}} i_{ \pm}(M) & =\min \left\{i_{ \pm}(A)+n, \quad i_{ \pm}(B)+m\right\}, \\
\min _{X \in \mathbb{C}^{m \times n}} i_{ \pm}(M) & =\max \left\{i_{ \pm}(A), \quad i_{ \pm}(B)\right\} .
\end{aligned}
$$

Substituting (2.26)-(2.29) into (2.22)-(2.25) and simplifying produces (2.18)-(2.21). Results (a)-(f) follow from (2.18)-(2.21) and Lemma 1.1.

The matrices $X$ satisfying (2.18)-(2.21), or equivalently satisfying (2.26)-(2.29), can be constructed respectively from the canonical forms of $A$ and $B$, as demonstrated in the proof of Theorem 2.1.

If the Hermitian matrix $A \in \mathbb{C}_{h}^{m \times m}$ in Theorem 2.3 is given with $r(A)=k<m$, then it can be decomposed by (2.6) as

$$
A=P^{*} D P
$$


where $D \in \mathbb{C}_{h}^{k \times k}$ and $P \in \mathbb{C}^{k \times m}$ satisfy $r(D)=r(P)=k$. In this case, $B-X^{*} A X$ can equivalently be expressed as

$$
B-X^{*} A X=B-X^{*} P^{*} D P X=B-Y^{*} D Y,
$$

where $Y=P X$. In this case, applying Theorem 2.3 to $B-Y^{*} D Y$ will yield the corresponding extremal values of the rank and inertia of $B-X^{*} A X$ with respect to $X$.

The inertia of rank 1 Hermitian perturbation of Hermitian matrix was considered in [17]. Now setting $A=\alpha I_{m}$ in Theorem 2.3, we obtain the following result on the rank and inertia of a Hermitian matrix with a Hermitian perturbation of arbitrary rank.

Corollary 2.4. Let $B \in \mathbb{C}_{h}^{n \times n}$ be given, and assume $\alpha>0$. Then

$$
\begin{aligned}
\max _{X \in \mathbb{C}^{m \times n}} r\left(B-\alpha X^{*} X\right) & =\min \{n, \quad m+r(B)\}, \\
\min _{X \in \mathbb{C}^{m \times n}} r\left(B-\alpha X^{*} X\right) & =\max \left\{i_{-}(B), \quad r(B)-m\right\}, \\
\max _{X \in \mathbb{C}^{m \times n}} i_{+}\left(B-\alpha X^{*} X\right) & =i_{+}(B), \\
\max _{X \in \mathbb{C}^{m \times n}} i_{-}\left(B-\alpha X^{*} X\right) & =\min \left\{n, \quad m+i_{-}(B)\right\}, \\
\min _{X \in \mathbb{C}^{m \times n}} i_{+}\left(B-\alpha X^{*} X\right) & =\max \left\{0, i_{+}(B)-m\right\}, \\
\min _{X \in \mathbb{C}^{m \times n}} i_{-}\left(B-\alpha X^{*} X\right) & =i_{-}(B) .
\end{aligned}
$$

3. Rank and inertia completions for an $n \times n$ block Hermitian matrix. Without much effort, the results in Theorem 2.1 can be extended to the block matrix in (1.2).

Theorem 3.1. Denote the matrix $M \in \mathbb{C}_{h}^{s \times s}$ in (1.2) as

$$
M=\left[\begin{array}{cccc}
A_{11} & X_{12} & \ldots & X_{1 n} \\
X_{12}^{*} & A_{22} & \ldots & X_{2 n} \\
\vdots & \vdots & \ddots & \vdots \\
X_{1 n}^{*} & X_{2 n}^{*} & \ldots & A_{n n}
\end{array}\right], \quad A_{j j} \in \mathbb{C}_{h}^{s_{j} \times s_{j}}, \quad j=1, \ldots, n
$$


where $X_{j k} \in \mathbb{C}^{s_{j} \times s_{k}}, j<k, j, k=1, \ldots, n$, are variable matrices, and $s=\sum_{j=1}^{n} s_{j}$. Then

$$
\max _{X_{j k} \in \mathbb{C}^{s_{j} \times s_{k}}, j<k} r(M)=\min \left\{s, 2\left(s-s_{1}\right)+r\left(A_{11}\right), \ldots, 2\left(s-s_{n}\right)+r\left(A_{n n}\right)\right\},
$$

$$
\min _{X_{j k} \in \mathbb{C}^{s_{j} \times s_{k}}, j<k} r(M)=\max \left\{i_{+}\left(A_{11}\right), \ldots, i_{+}\left(A_{n n}\right)\right\}+\max \left\{i_{-}\left(A_{11}\right), \ldots, i_{-}\left(A_{n n}\right)\right\},
$$

$$
\max _{X_{j k} \in \mathbb{C}^{s_{j} \times s_{k}}, j<k} i_{ \pm}(M)=\min \left\{s-s_{1}+i_{ \pm}\left(A_{11}\right), \ldots, s-s_{n}+i_{ \pm}\left(A_{n n}\right)\right\},
$$

$$
\min _{X_{j k} \in \mathbb{C}^{s_{j} \times s_{k}}, j<k} i_{ \pm}(M)=\max \left\{i_{ \pm}\left(A_{11}\right), \ldots, i_{ \pm}\left(A_{n n}\right)\right\} .
$$

Hence,

(a) There exist $X_{j k}, j<k, j, k=1, \ldots, n$, such that $M$ is nonsingular if and only if

$$
r\left(A_{11}\right) \geq 2 s_{1}-s, \ldots, r\left(A_{n n}\right) \geq 2 s_{n}-s .
$$

(b) There exist $X_{j k}, j<k, j, k=1, \ldots, n$, such that $M>0(M<0)$ if and only if

$$
A_{11}>0, \ldots, A_{n n}>0 \quad\left(A_{11}<0, \ldots, A_{n n}<0\right) .
$$

(c) There exist $X_{j k}, j<k, j, k=1, \ldots, n$, such that $M \geq 0(M \leq 0)$ if and only if

$$
A_{11} \geq 0, \ldots, A_{n n} \geq 0 \quad\left(A_{11} \leq 0, \ldots, A_{n n} \leq 0\right) .
$$

Proof. Rewrite the matrix $M$ in (3.1) as

$$
M=\left[\begin{array}{cc}
M_{1} & X_{n} \\
X_{n}^{*} & A_{n n}
\end{array}\right],
$$

where $M_{1}$ is a block matrix of order $n-1$ with the same structure as $M$. Applying (2.2) to this $M$ gives

$$
\max _{X_{n}} r(M)=\min \left\{s, 2\left(s-s_{n}\right)+r\left(A_{n n}\right), 2 s_{n}+r\left(M_{1}\right)\right\} .
$$

Thus (3.2) follows by induction on the matrix $M_{1}$ in (3.6). Applying (2.4) to (3.6) gives

$$
\max _{X_{n}} i_{ \pm}(M)=\min \left\{s-s_{n}+i_{ \pm}\left(A_{n n}\right), \quad s_{n}+i_{ \pm}\left(M_{1}\right)\right\} .
$$


Thus (3.4) follows by induction on the matrix $M_{1}$ in (3.6).

Applying (2.3) to (3.6) gives

$$
\min _{X_{n}} r(M)=\max \left\{i_{+}\left(M_{1}\right), i_{+}\left(A_{n n}\right)\right\}+\max \left\{i_{-}\left(M_{1}\right), \quad i_{-}\left(A_{n n}\right)\right\} .
$$

Because $M_{1}$ occurs twice in (3.9), we cannot derive the minimal values of $i_{+}\left(M_{1}\right)$ and $i_{-}\left(M_{1}\right)$ separately, and thus we cannot go further along (3.9). In such a case, we have to use the following canonical decomposition of $A_{j j}$, as well as the corresponding decomposition of $X_{j k}$ in (3.1):

$$
W_{j} A_{j j} W_{j}^{*}=\left[\begin{array}{ccc}
I_{p_{j}} & 0 & 0 \\
0 & -I_{q_{j}} & 0 \\
0 & 0 & 0
\end{array}\right], \quad W_{j} X_{j k} W_{k}^{*}=\left[\begin{array}{ccc}
Z_{j k}^{(11)} & Z_{j k}^{(12)} & Z_{j k}^{(13)} \\
Z_{j k}^{(21)} & Z_{j k}^{(22)} & Z_{j k}^{(23)} \\
Z_{j k}^{(31)} & Z_{j k}^{(32)} & Z_{j k}^{(33)}
\end{array}\right]
$$

where $p_{j}=i_{+}\left(A_{j j}\right), q_{j}=i_{-}\left(A_{j j}\right), W_{j}$ is a nonsingular matrix, $Z_{j k}^{(s t)}$ is an arbitrary matrix of appropriate size, $j<k$ and $j, k=1, \ldots, n$, and $s, t=1,2,3$. Denote $W=\operatorname{diag}\left(W_{1}, \ldots, W_{n}\right)$. Then

$$
r(M)=r\left(W M W^{*}\right)=r\left[\begin{array}{cccc}
W_{1} A_{11} W_{1}^{*} & W_{1} X_{12} W_{2}^{*} & \ldots & W_{1} X_{1 n} W_{n}^{*} \\
W_{2} X_{12}^{*} W_{1}^{*} & W_{2} A_{22} W_{2}^{*} & \ldots & W_{2} X_{2 n} W_{n}^{*} \\
\vdots & \vdots & \ddots & \vdots \\
W_{n} X_{1 n}^{*} W_{1}^{*} & W_{n} X_{2 n}^{*} W_{2}^{*} & \ldots & W_{n} A_{n n} W_{n}^{*}
\end{array}\right] .
$$

Removing the third block rows and columns in all $W_{j} X_{j k} W_{k}^{*}$ s on the right-hand side of (3.11) leads to the following rank inequality

$$
r(M) \geq r\left[\begin{array}{cccc}
\operatorname{diag}\left(I_{p_{1}},-I_{q_{1}}\right) & Z_{12} & \ldots & Z_{1 n} \\
Z_{12}^{*} & \operatorname{diag}\left(I_{p_{2}},-I_{q_{2}}\right) & \ldots & Z_{2 n} \\
\vdots & \vdots & \ddots & \vdots \\
Z_{1 n}^{*} & Z_{2 n}^{*} & \ldots & \operatorname{diag}\left(I_{p_{n}},-I_{q_{n}}\right)
\end{array}\right]
$$

where $Z_{j k}=\left[\begin{array}{cc}Z_{j k}^{(11)} & Z_{j k}^{(12)} \\ Z_{j k}^{(21)} & Z_{j k}^{(22)}\end{array}\right], j<k$ and $j, k=1, \ldots, n$. Comparing the block matrix in (3.12) with its submatrices gives rise to the following rank inequalities

$$
\begin{aligned}
r(M) & \geq p_{j}+q_{j}, \quad j=1, \ldots, n \\
r(M) \geq r\left[\begin{array}{cc}
I_{p_{j}} & Z_{j k}^{(12)} \\
\left(Z_{j k}^{(12)}\right)^{*} & -I_{q_{k}}
\end{array}\right] & =r\left[\begin{array}{cc}
I_{p_{j}} & 0 \\
0 & -I_{q_{k}}-\left(Z_{j k}^{(12)}\right)^{*} Z_{j k}^{(12)}
\end{array}\right] \\
& =p_{j}+r\left[I_{q_{k}}+\left(Z_{j k}^{(12)}\right)^{*} Z_{j k}^{(12)}\right] \\
& =p_{j}+q_{k}, \quad j<k \text { and } j, k=1, \ldots, n .
\end{aligned}
$$


These inequalities indicate the right-hand side of (3.3) is a lower bound for $r(M)$. Without loss of generality, we assume that

$$
p_{1} \geq \max \left\{p_{2}, \ldots, p_{n}\right\} \text { and } q_{1} \geq \max \left\{q_{2}, \ldots, q_{n}\right\},
$$

and set

$$
W_{j} X_{j k} W_{k}^{*}=\left[\begin{array}{ccc}
Z_{j k}^{(11)} & 0 & 0 \\
0 & Z_{j k}^{(22)} & 0 \\
0 & 0 & 0
\end{array}\right], \quad j<k \text { and } j, k=1, \ldots, n
$$

in (3.10). Then the rank of the corresponding matrix $M$ in (3.11) can be written as the sum

$$
\begin{aligned}
& r(M)=r\left[\begin{array}{cccc}
I_{p_{1}} & Z_{12}^{(11)} & \ldots & Z_{1 n}^{(11)} \\
\left(Z_{12}^{(11)}\right)^{*} & I_{p_{2}} & \ldots & Z_{2 n}^{(11)} \\
\vdots & \vdots & \ddots & \vdots \\
\left(Z_{1 n}^{(11)}\right)^{*} & \left(Z_{2 n}^{(11)}\right)^{*} & \ldots & I_{p_{n}}
\end{array}\right]+r\left[\begin{array}{cccc}
-I_{q_{1}} & Z_{12}^{(22)} & \ldots & Z_{1 n}^{(22)} \\
\left(Z_{12}^{(22)}\right)^{*} & -I_{q_{2}} & \ldots & Z_{2 n}^{(22)} \\
\vdots & \vdots & \ddots & \vdots \\
\left(Z_{1 n}^{(22)}\right)^{*} & \left(Z_{2 n}^{(22)}\right)^{*} & \ldots & -I_{q_{n}}
\end{array}\right] \\
& =r\left[\begin{array}{cccc}
I_{p_{1}} & 0 & \ldots & 0 \\
0 & I_{p_{2}}-\left(Z_{12}^{(11)}\right)^{*} Z_{12}^{(11)} & \ldots & Z_{2 n}^{(11)}-\left(Z_{12}^{(11)}\right)^{*} Z_{1 n}^{(11)} \\
\vdots & \vdots & \ddots & \vdots \\
0 & \left(Z_{2 n}^{(11)}\right)^{*}-\left(Z_{1 n}^{(11)}\right)^{*} Z_{12}^{(11)} & \ldots & I_{p_{n}}-\left(Z_{1 n}^{(11)}\right)^{*} Z_{1 n}^{(11)}
\end{array}\right] \\
& +r\left[\begin{array}{cccc}
-I_{q_{1}} & 0 & \ldots & 0 \\
0 & -I_{q_{2}}+\left(Z_{12}^{(22)}\right)^{*} Z_{12}^{(22)} & \ldots & Z_{2 n}^{(22)}+\left(Z_{12}^{(22)}\right)^{*} Z_{1 n}^{(22)} \\
\vdots & \vdots & \ddots & \vdots \\
0 & \left(Z_{2 n}^{(22)}\right)^{*}+\left(Z_{1 n}^{(22)}\right)^{*} Z_{12}^{(22)} & \ldots & -I_{q_{n}}+\left(Z_{1 n}^{(22)}\right)^{*} Z_{1 n}^{(22)}
\end{array}\right] .
\end{aligned}
$$

Under (3.15), there exist $Z_{12}^{(11)}, \ldots, Z_{1 n}^{(11)}$ and $Z_{12}^{(22)}, \ldots, Z_{1 n}^{(22)}$ such that

$$
\begin{aligned}
& \left(Z_{12}^{(11)}\right)^{*} Z_{12}^{(11)}=I_{p_{2}}, \ldots,\left(Z_{1 n}^{(11)}\right)^{*} Z_{1 n}^{(11)}=I_{p_{n}}, \\
& \left(Z_{12}^{(22)}\right)^{*} Z_{12}^{(22)}=I_{q_{2}}, \ldots,\left(Z_{1 n}^{(11)}\right)^{*} Z_{1 n}^{(11)}=I_{q_{n}},
\end{aligned}
$$

say $\left(Z_{1 k}^{(11)}\right)^{*}=\left[I_{p_{k}}, 0\right]$ and $\left(Z_{1 k}^{(22)}\right)^{*}=\left[I_{q_{k}}, 0\right], k=2, \ldots, n$. Also set

$$
Z_{j k}^{(11)}=\left(Z_{1 j}^{(11)}\right)^{*} Z_{1 k}^{(11)} \text { and } Z_{j k}^{(22)}=-\left(Z_{1 j}^{(22)}\right)^{*} Z_{1 k}^{(22)}, \quad j<k \text { and } j, k=1, \ldots, n \text {. }
$$

Then (3.16) becomes $r(M)=p_{1}+q_{1}$, which satisfies (3.3).

Applying (2.5) to (3.6) gives

$$
\min _{X_{n}} i_{ \pm}(M)=\max \left\{i_{ \pm}\left(M_{1}\right), \quad i_{ \pm}\left(A_{n n}\right)\right\} .
$$


Thus (3.5) follows by induction on the matrix $M_{1}$ in (3.6). Results (a), (b) and (c) follow from (3.2)-(3.5) and Lemma 1.1.

The quantities on the right-hand sides of (3.4) and (3.5), as upper and lower bounds for the inertia of $M$ in (3.1), were given in Theorem 2.1 and Lemma 3.1 of [1].

4. Concluding remarks and open problems. The author provided in this paper some procedures of completing the partial block Hermitian matrices in (2.1) and (3.1) such that the resulting matrices have the maximal and minimal possible ranks and inertias, respectively. From these extremal ranks and inertias, the author derived necessary and sufficient conditions for the partial block Hermitian matrices in (2.1) and (3.1) to be invertible and positive (negative) definite, respectively. It is expected that the results obtained in this paper can be used to solve completion problems on ranks, inertias, invertibility and definiteness of some other types of partial block Hermitian matrices.

More matrix completion problems associated with the ranks and inertias of the partial block Hermitian matrices in (2.1) and (3.1) can be proposed. For instance, for any integer between the maximal and minimal values of the ranks and inertias in Theorems 2.1 and 3.1, consider how to choose the variable matrices in (2.1) and (3.1) such that the rank and inertia of the completed matrix have this given integer, respectively.

Note that a partial square matrix of order $m$ has an invertible completion if and only if the maximal rank of the partial matrix is equal to $m$. Hence it would be of interest to consider under Theorems 2.1(a) and 3.1(a) the following inverse completion problems

$$
\begin{gathered}
{\left[\begin{array}{cc}
A & ? \\
?^{*} & B
\end{array}\right]^{-1}=\left[\begin{array}{cc}
C & ? \\
?^{*} & D
\end{array}\right], \quad\left[\begin{array}{cc}
A & ? \\
?^{*} & B
\end{array}\right]^{-1}=\left[\begin{array}{cc}
? & P \\
P^{*} & ?
\end{array}\right],} \\
{\left[\begin{array}{cccc}
A_{11} & ? & \ldots & ? \\
?^{*} & A_{22} & \ldots & ? \\
\vdots & \vdots & \ddots & \vdots \\
?^{*} & ?^{*} & \ldots & A_{n n}
\end{array}\right]=\left[\begin{array}{cccc}
? & B_{12} & \ldots & B_{1 n} \\
B_{12}^{*} & ? & \ldots & B_{2 n} \\
\vdots & \vdots & \ddots & \vdots \\
B_{1 n}^{*} & B_{2 n}^{*} & \ldots & ?
\end{array}\right] .}
\end{gathered}
$$

Matrix completion problems are often considered under some restrictions to unspecified entries in a given partial matrix. Some previous work on the inertias of the block matrices in (2.1) and (3.1) with restrictions to the specified or unspecified entries can be found, e.g., in [8]-[10] and [17]. As a continuation of the work in the previous sections, it is of interest to derive maximal and minimal ranks and inertias 
of the Hermitian matrices under various restrictions, such as,

$$
\begin{aligned}
& {\left[\begin{array}{cc}
A & X \\
X^{*} & B
\end{array}\right] \quad \text { subject to } X=A Y B \text { or } r(X)=k \leq \min \{m, n\},} \\
& B-X^{*} A X \text { subject to } X^{*} X=I_{n} \text { or } X X^{*}=I_{m} .
\end{aligned}
$$

Another valuable work on the block Hermitian matrix in (2.1) as well as the matrix expressions $B \pm X^{*} A X$ is to solve the following rank/inertia additivity/subtractivity equations

$$
\begin{aligned}
& r\left[\begin{array}{cc}
A & X \\
X^{*} & B
\end{array}\right]=r(A)+r\left[\begin{array}{cc}
0 & X \\
X^{*} & B
\end{array}\right], \quad i_{ \pm}\left[\begin{array}{cc}
A & X \\
X^{*} & B
\end{array}\right]=i_{ \pm}(A)+i_{ \pm}\left[\begin{array}{cc}
0 & X \\
X^{*} & B
\end{array}\right], \\
& r\left[\begin{array}{cc}
A & X \\
X^{*} & B
\end{array}\right]=r(A)+r(B)+2 r(X), \quad i_{ \pm}\left[\begin{array}{cc}
A & X \\
X^{*} & B
\end{array}\right]=i_{ \pm}(A)+i_{ \pm}(B)+r(X), \\
& r\left(B \pm X^{*} A X\right)=r(B) \pm r\left(X^{*} A X\right), \quad i_{ \pm}\left(B \pm X^{*} A X\right)=i_{ \pm}(B) \pm i_{ \pm}\left(X^{*} A X\right) .
\end{aligned}
$$

These further developments are beyond the scope of the present paper and will be the subjects of separate studies.

\section{Acknowledgements}

The author is grateful to an anonymous referee and the handling editor for their helpful suggestions and constructive comments on the current version of this paper. The author thanks Professor Fuzhen Zhang for helpful suggestions and comments on an earlier version of this paper.

\section{REFERENCES}

[1] B.E. Cain. The inertia of a Hermitian matrix having prescribed diagonal blocks. Linear Algebra Appl., 37:173-180, 1981.

[2] B.E. Cain and E.M. de Sá. The inertia of a Hermitian matrix having prescribed complementary principal submatrices. Linear Algebra Appl., 37:161-171, 1981.

[3] B.E. Cain and E.M. de Sá. The inertia of Hermitian matrices with a prescribed $2 \times 2$ block decomposition. Linear and Multilinear Algebra, 31:119-130, 1992.

[4] D. Chu, Y.S. Hung and H.J. Woerdeman. Inertia and rank characterizations of some matrix expressions. SIAM. J. Matrix Anal. Appl., 31:1187-1226, 2009.

[5] N. Cohen and J. Dancis. Maximal rank Hermitan completions of partially specified Hermitian matrices. Linear Algebra Appl., 244:265-276, 1996.

[6] N. Cohen and J. Dancis. Inertias of block band matrix completions. SIAM J. Matrix Anal. Appl., 19:583-612, 1998.

[7] N. Cohen, C.R. Johnson. L. Rodman and H.J. Woerdeman. Rank completions of partial matrices. Operator Theory: Adv. Appl., 40:165-185, 1989. 
[8] C.M. da Fonseca. The inertia of certain Hermitian block matrices. Linear Algebra Appl., 274:193-210, 1998.

[9] C.M. da Fonseca. The inertia of Hermitian block matrices with zero main diagonal. Linear Algebra Appl., 311:153-160, 2000.

[10] C.M. da Fonseca. A conjecture about the inertia of Hermitian matrices. Math. Inequal. Appl., 7:497-503, 2004.

[11] J. Dancis. The possible inertias for a Hermitian matrix and its principal submatrices. Linear Algebra Appl., 85:121-151, 1987.

[12] J. Dancis. Invertible completions of band matrices. Linear Algebra Appl., 150:125-138, 1991.

[13] J. Dancis. Poincaré's inequalities and Hermitian completions of certain partial matrices. Linear Algebra Appl., 167:219-225, 1992.

[14] J. Dancis. Positive semidefinite completions of partial Hermitian matrices. Linear Algebra Appl., 175:97-114, 1992.

[15] J.F. Geelen. Maximum rank matrix completion. Linear Algebra Appl., 288:211-217, 1999.

[16] I. Gohberg, M.A. Kaashoek and F. van Schagen. Partially Specified Matrices and Operators: Classification, Completion, Applications. Birkhauser, 1995.

[17] D.A. Gregory, B. Heyink and K.N.V. Meulen. Inertia and biclique decompositions of joins of graphs. J. Combin. Theory Ser. B, 88:135-151, 2003.

[18] D.A. Gregory, B.L. Shader and V.L. Watts. Biclique decompositions and Hermitian rank. Linear Algebra Appl., 292:267-280, 1999.

[19] J. Grone, C.R. Johnson, E.M. de Sá and H. Wolkowitz. Positive definite completions of partial Hermitian matrices. Linear Algebra Appl., 58:109-124, 1984.

[20] N.J.A. Harvey, D.R. Karger and S. Yekhanin. The complexity of matrix completion. In: Proceedings of the Seventeenth Annual ACM-SIAM Symposium on Discrete Algorithm. Association for Computing Machinery, New York, pp. 1103-1111, 2006.

[21] E.V. Haynsworth. Determination of the inertia of a partitioned Hermitian matrix. Linear Algebra Appl., 1:73-81, 1968.

[22] E.V. Haynsworth and A.M. Ostrowski. On the inertia of some classes of partitioned matrices. Linear Algebra Appl., 1:299-316, 1968.

[23] C.R. Johnson and L. Rodman. Inertial possibilities for completions of partial Hermitian matrices. Linear and Multilinear Algebra, 16:179-195, 1984.

[24] C.R. Johnson and G.T. Whitney. Minimum rank completions. Linear and Multilinear Algebra, 28: 271-273, 1991.

[25] M. Laurent. Matrix completion problems. In: The Encyclopedia of Optimization (C. Floudas and P. Pardalos, eds.), Vol. III, Kluwer, pp. 221-229, 2001.

[26] J.H. Maddocks. Restricted quadratic forms, inertia theorems and the Schur complement. Linear Algebra Appl., 108:1-36, 1988.

[27] M. Mahajan and J. Sarma. On the complexity of matrix rank and rigidity. Lecture Notes in Computer Science, Vol. 4649, Springer, pp. 269-280, 2007.

[28] K. Takahashi. Invertible completions of operator matrices. Integ. Equ. Oper. Theory, 21:355-361, 1995.

[29] Y. Tian. Completing block matrices with maximal and minimal ranks. Linear Algebra 
Appl., 321: 327-345, 2000.

[30] Y. Tian. The minimum rank of a $3 \times 3$ partial block matrix. Linear and Multilinear Algebra, 50:125-131, 2002.

[31] Y. Tian and Y. Takane. The inverse of any two-by-two nonsingular partitioned matrix and three matrix inverse completion problems. Comput. Math. Appl., 57:1294-1304, 2009.

[32] H.J. Woerdeman. Minimal rank completions for block matrices. Linear Algebra Appl., 121:105-122, 1989.

[33] H.J. Woerdeman. Toeplitz minimal rank completions. Linear Algebra Appl., 202:267$278,1994$.

[34] H.J. Woerdeman. Minimal rank completions of partial banded matrices. Linear Multilinear Algebra, 36:59-68, 1993.

[35] H.J. Woerdeman. Hermitian and normal completions. Linear and Multilinear Algebra, 42:239-280, 1997. 